\title{
Gemcitabine combined with an engineered oncolytic vaccinia virus exhibits a synergistic suppressive effect on the tumor growth of pancreatic cancer
}

\author{
WANYUAN CHEN $^{1 *}$, WEIMIN FAN ${ }^{2 *}$, GUOQING RU $^{1}$, FANG HUANG $^{1}$, XIAMING LU $^{1}$, \\ XIN ZHANG ${ }^{1}$, XIAOZHOU MOU ${ }^{3,4}$ and SHIBING WANG ${ }^{3,4}$
}

\author{
${ }^{1}$ Department of Pathology, Zhejiang Provincial People's Hospital, People's Hospital of Hangzhou Medical College, Hangzhou, \\ Zhejiang 310014; ${ }^{2}$ The Second Clinical Medical College, Zhejiang Chinese Medical University, Hangzhou, \\ Zhejiang 310053; ${ }^{3}$ Clinical Research Institute, Zhejiang Provincial People's Hospital, People's Hospital \\ of Hangzhou Medical College; ${ }^{4}$ Key Laboratory of Tumor Molecular Diagnosis and Individualized \\ Medicine of Zhejiang Province, Hangzhou, Zhejiang 310014, P.R. China
}

Received February 4, 2018; Accepted October 3, 2018

DOI: $10.3892 /$ or.2018.6817

\begin{abstract}
Pancreatic cancer (PC) is a lethal solid malignancy with resistance to traditional chemotherapy. Recently, considerable studies have demonstrated the ubiquitous antitumor properties of gene therapy mediated by the oncolytic vaccinia virus. The second mitochondrial-derived activator of caspase (Smac) has been identified as an innovative tumor suppressor that augments the chemosensitivity of cancer cells. However, the therapeutic value of oncolytic vaccinia virus (oVV)-mediated Smac gene transfer in pancreatic cancer is yet to be elucidated. In the present study, oncolytic vaccinia virus expressing Smac (second mitochondrial-derived activator of caspase) (oVV-Smac) was used to examine its beneficial value when used alone or with gemcitabine in pancreatic cancer in vitro and in vivo. The expression of Smac was evaluated by western blot analysis and quantitative polymerase chain reaction, oVV-Smac cytotoxicity by MTT assay, and apoptosis by flow cytometry and western blot analysis. Furthermore, the inhibitory effect of oVV-Smac combined with gemcitabine was also evaluated. The results indicated that oVV-Smac achieved high levels of Smac, greater cytotoxicity, and potentiated apoptosis. Moreover, co-treatment with oVV-Smac and gemcitabine resulted in a synergistic effect in vitro and in vivo. Therefore, our findings advance oVV-Smac as a potential therapeutic
\end{abstract}

Correspondence to: Dr Shibing Wang, Clinical Research Institute, Zhejiang Provincial People's Hospital, People's Hospital of Hangzhou Medical College, 158 Shangtang Road, Hangzhou, Zhejiang 310014, P.R. China

E-mail: wangshibing@hmc.edu.cn

*Contributed equally

Key words: oncolytic vaccinia virus, Smac, gemcitabine, pancreatic cancer, drug resistance candidate in pancreatic cancer and indicated the synergistic effects of co-treatment with oVV-Smac and gemcitabine.

\section{Introduction}

Pancreatic cancer is an aggressive tumor type, with the highest mortality rate and poorest long-term prognosis of all cancer types. Since the pancreas is located in a deep retroperitoneal site and no specific symptoms are observable at early stages of pancreatic cancer, the diagnosis at a surgically resectable stage is difficult $(1,2)$. Pancreatic cancer is a highly lethal disease for which limited therapeutic options are available, which often combines gemcitabine with other chemotherapeutics $(3,4)$. However, these chemotherapeutic agents are highly toxic and lack therapeutic efficacy. In particular, clinical beneficial responses to gemcitabine are only observed in approximately $25 \%$ of cases, and, owing to long-term tolerance, this limited therapeutic efficacy rapidly declines resulting in a median overall survival of 6 months $(5,6)$. Therefore, a novel strategy is needed to optimize the efficacy of gemcitabine in treating pancreatic cancer.

Oncolytic viruses (OVs) are native or recombinant viruses which can selectively kill cancer cells and associated stromal cells directly by oncolysis, indirectly by immune mediated clearance of cancer cells, or targeting of tumor vasculature. Vaccinia virus is highly immunogenic and has properties that make it an ideal oncolytic immunotherapy vector (7). Preclinical murine studies have demonstrated significant antitumor efficacy and systemic antitumor immunity, using a tumor-selective oncolytic vaccinia virus expressing immunogenic transgenes (8-11). An oncolytic vaccinia virus armed with GM-CSF (Pexa-Vec) was associated with a $15 \%$ objective response rate in patients with advanced hepatocellular carcinoma in a randomized phase II clinical trial (11). The oncolytic vaccinia virus (oVV), which selectively replicates in cancer cells, is a promising alternative to conventional therapy for cancer treatment and has been extensively examined in clinical trials (11-13). In addition to its safety profile, one of 
the most important advantages is that the $\sim 200 \mathrm{~kb}$ genome of vaccinia virus enables the insertion of foreign genes up to $25 \mathrm{~kb}$ in length. Furthermore, the oVV has a broad range of host cells, high capacity of transgene expression, and its activity is unrestrained by hypoxia $(12,14)$. Indeed, oVV-based gene therapy has been investigated in a range of tumor types, such as myeloma, pancreatic carcinoma, hepatocellular carcinoma, and gastric carcinoma, and has been reported to induce significant growth suppression with relatively limited side effects, indicating that oVV is a promising vector for cancer gene therapy (15-17).

The second mitochondrial-derived activator of caspase (Smac) is released from mitochondria into the cytosol during the process of apoptosis $(18,19)$. It has been previously demonstrated that the release of Smac is critical for apoptosis induced by anti-myeloma agents (20). Furthermore, alterations in Smac release contribute to drug resistance in cancer cells. Our previous study demonstrated the role of Smac in promoting apoptosis in pancreatic cancer cells by decreasing the expression of the inhibitor of apoptosis proteins (IAPs) (21). Based on these previous findings, the regulation of Smac expression seems a promising therapy in pancreatic cancer. We previously demonstrated that oVV-expressing Smac exerted potent antitumor efficacy in hepatocellular carcinoma (22) and Smac-armed oncolytic adenovirus significantly inhibited pancreatic cancer and multiple myeloma growth $(23,24)$. However, the therapeutic efficacy of oVV-mediated Smac gene therapy in human pancreatic cancer is yet to be elucidated.

This present study aimed at investigating the antitumor properties of oVV-Smac both alone or combined with gemcitabine in pancreatic cancer cells and xenograft mouse models.

\section{Materials and methods}

Cell lines and virus. The human pancreatic cancer cell lines SW1990, BXPC-3 and PANC-1 were purchased from the Cell Bank of the Type Culture Collection of the Chinese Academy of Sciences (Shanghai, China). SW1990/GEM is gemcitabine-resistant SW1990 cell line, which was retained in our laboratory. All the cell lines were authenticated by short-tandem repeat profiling and cultured in $\mathrm{Gibco}^{\mathrm{TM}}$ Dulbecco's modified Eagle's medium (DMEM) Thermo Fisher Scientific, Inc., Waltham, MA, USA) supplemented with $10 \%$ heat inactivated fetal bovine serum (FBS; Thermo Fisher Scientific, Inc.). Cells were incubated in a $5 \% \mathrm{CO}_{2}$ humidified incubator at $37^{\circ} \mathrm{C}$. Construction and production of recombinant oncolytic vaccinia virus oVV-Smac and oVV were previously described (24). The Smac gene was inserted into the thymidine kinase (TK) region, disrupting the function of TK. Deletion of the TK gene inhibits viral replication in normal, non-dividing cells (25). However, cancer cells have a high concentration of functional nucleotides that enables oVV replication to occur in the absence of viral TK. Therefore, disruption of TK results in selective replication of the oVV in tumor cells. The T7 promoter was inserted before the exogenous genes to initiate their expression, and the gpt gene works as a screen gene engineered behind the exogenous genes. The whole expression cassette was constructed into the $\mathrm{pCB}$ vector, which is a shuttle plasmid for vaccinia virus packaging kindly provided by academician Xinyuan Liu (Shanghai Institutes for Biological Sciences, Chinese Academy of Sciences, Shanghai, China). Each recombinant vaccinia virus was isolated through three rounds of plaque purification in 293 cells and purified by ultracentrifugation in a cesium chloride gradient. Moreover, virus titers were determined by TCID50 assay in 293 cells. Cells were infected with vaccinia virus at different doses at $37^{\circ} \mathrm{C}$ in a humidified atmosphere containing $5 \% \mathrm{CO}_{2}$.

Cell viability assay. PANC-1, SW1990 and BxPC-3 cells were dispensed in 96-well culture plates at a density of $5 \times 10^{3}$ cells/well. After attachment, cells were infected with oVV, oVV-Smac with or without gemcitabine at given concentrations and times. The medium added together with PBS was used as a blank control. The cell survival rate was evaluated by a standard 3-(4,5-dimethylthiazol-2-yl)-2,5-diphenyltetrazolium bromide (MTT) assay (Sigma-Aldrich; Merck KGaA, Darmstadt, Germany), Medium was removed and fresh medium containing MTT $(5 \mathrm{mg} / \mathrm{ml})$ was added to each well. The cells were incubated at $37^{\circ} \mathrm{C}$ for $4 \mathrm{~h}$, and after the supernatant was drawn off of each well carefully and an equal volume $(150 \mu \mathrm{l})$ of DMSO was added to each well and mixed thoroughly on a concentrating table for $10 \mathrm{~min}$. The absorbance of the plates was read at $595 \mathrm{~nm}$ with a GENios model DNA Expert Microplate Reader (Tecan Group, Ltd., Mannedorf, Switzerland). For combination index plots, CI is expressed as the $\log _{10}(\mathrm{CI}) \pm 1.96 \mathrm{SD}$, and the $95 \%$ confidence intervals (CIs) are shown where estimable, with the use of the algebraic approximation algorithm of the CalcuSyn program (Biosoft, Cambridge, UK). In the present study, CI values were calculated over a scope of levels of growth inhibition (GI) from 20 to $80 \%$ of the fraction affected.

Western blot analysis. Cells were harvested in lysis buffer (Beyotime Institute of Biotehnology, Jiangsu, China) containing 1\% Complete Mini-Protease Inhibitor Cocktail (Roche Diagnostics, Basel, Switzerland) and $5 \mathrm{mM} \mathrm{NaF}$. Protein extractions were quantified using the BCA kit (Thermo Fisher Scientific, Inc.) and heated for $10 \mathrm{~min}$ at $100^{\circ} \mathrm{C}$. Protein $(30 \mu \mathrm{g})$ was resolved on $12 \%$ SDS-PAGE and transferred to nitrocellulose membranes (Merk Millipore, Darmstadt, Germany). After blocking for $1 \mathrm{~h}$ at $37^{\circ} \mathrm{C}$, the membranes were immunoblotted with different antibodies overnight at $4^{\circ} \mathrm{C}$. Antibodies against Smac (dilution 1:1,000; cat. no. ab8114), vaccinia virus (dilution 1:500; cat. no. ab19970), GAPDH (dilution 1:2,000; cat. no. ab128915) were purchased from Abcam (Shanghai, China). Antibodies against caspase-8 (dilution 1:1,000; cat. no. MABC1606), caspase-9 (dilution 1:1,000; cat. no. MAB4709), caspase-3 (dilution 1:1,000; cat. no. AB3623), PARP (dilution 1:500; cat. no. AB16661), XIAP (dilution 1:1,000; cat. no. 07735), cIAP-1 (dilution 1:1,000; cat. no. ABC448), cIAP-2 (dilution 1:1,000; cat. no. AB3615), survivin (dilution 1:1,000; cat. no. MAB4617), livin (dilution 1:1,000; cat. no. ABC97), P-gp (dilution 1:500; cat. no. ABN455) and MDR1 (dilution 1:1,000; cat. no. MAB4162) were purchased from EMD Millipore Corp. (Billerica, MA, USA). Membranes were then washed with TBST and incubated with HRP-conjugated goat anti-rabbit (dilution 1:5,000; cat. no. HA1001) or anti-mouse antibody (dilution 1:5,000; cat. no. HA1006; both from for 
Huabio, Hangzhou, China) for $1 \mathrm{~h}$ at room temperature. Finally, blots were detected using ChemiDoc ${ }^{\mathrm{TM}}$ MP Imaging System (Bio-Rad Laboratories) with a Super Enhanced chemiluminescence detection kit (Applygen Technologies, Inc., Beijing, China).

Flow cytometric analysis. Cells infected with oncolytic vaccinia viruses and/or gemcitabine were trypsinized and washed once with complete medium. Aliquots of cells $\left(5 \times 10^{5}\right)$ were resuspended in $500 \mathrm{ml}$ of binding buffer and stained with fluorescein isothiocyanate (FITC)-labeled Annexin V and propidium iodide (PI) (BioVision, Inc., Palo Alto, CA, USA) according to the manufacturer's instructions. Cell apoptosis and cell cycle were examined using FACS (FACStar cytofluorometer; BD Biosciences, San Jose, CA, USA).

Real-time quantitative PCR. RNA was extracted with Invitrogen $^{\mathrm{TM}}$ Trizol reagent (Thermo Fisher Scientific, Inc.) following the manufacturer's instructions. cDNA was generated using the PrimeScript RT reagent kit (Takara Bio, Inc., Tokyo, Japan). The qPCR reactions were conducted in a total volume of $20 \mu \mathrm{l}$ by using the following procedure: 1 cycle at $95^{\circ} \mathrm{C}(10 \mathrm{~min})$, then $60^{\circ} \mathrm{C}(30 \mathrm{sec})$, followed by 39 cycles at $95^{\circ} \mathrm{C}(10 \mathrm{sec})$ and $60^{\circ} \mathrm{C}(30 \mathrm{sec})$. PCR amplicons were determined based on SYBR-Green I detection (Roche Diagnostic, Indianapolis, IN, USA), and the authenticity was certified by melting curve analysis. Quantitative PCR was operated using the CFX-96 qPCR system and iQ SYBR-Green SuperMix (Bio-Rad Laboratories). Relative gene expression was determined via the $2^{\Delta \Delta \mathrm{Ct}}$ method. The primers used are as follows: Smac, 5'-GGAAGATCTCCTCCTGCATCC-3' (forward) and 5'-CCGCTTAAGATACCGCTCGAG-3' (reverse); GAPDH, 5'-CTTTGGTATCGTGGAAGGACTC-3' (forward) and 5'-GTAGAGGCAGGGGATGATGTTCT-3' (reverse).

Animal experiments. All animal experiments were approved by the Institutional Animal Care and Use Committee, Zhejiang Provincial People's Hospital and all procedures were in accordance to the Guide for the Care and Use of Laboratory Animals (National Academies Press, Washington, DC). One hundred female BALB/c nude mice (4- to 5-weeks old, $20 \mathrm{~g}$ ) were purchased from the Shanghai Experimental Animal Center (Shanghai, China). All 100 mice were housed in a specific pathogen-free environment, in which the temperature was maintained at $26-28^{\circ} \mathrm{C}$, the humidity was $40-60 \%$, and the daily light was maintained for $10 \mathrm{~h}(14 \mathrm{~h}$ without light). Ventilation was ensured 10 to 15 times per hour. When the mouse tumor reached a diameter of $2.0 \mathrm{~cm}$ or a volume of $2.5 \mathrm{~cm}^{3}$, or all mice in the PBS group died, we stopped the mouse experiment. The sensitive SW1990 cells or SW1990/GEM cells were injected subcutaneously into the lower right flank of female nude mice and the tumor xenograft model was established. Each group was composed of at least 8 animals and tumor growth was monitored and measured for every 4 days with a Vernier caliper. Tumor volume (V) was calculated according to the formula: $\mathrm{V}\left(\mathrm{mm}^{3}\right)=1 / 2 \mathrm{x}$ length $(\mathrm{mm}) \mathrm{x}$ width $(\mathrm{mm})^{2}$. Once the subcutaneous tumors reached $\sim 100 \mathrm{~mm}^{3}$, the nude mice were divided into 5 groups ( 8 mice in each group) randomly. Subsequently, mice were injected with gemcitabine, oVV, oVV-Smac, gemcitabine plus oVV-Smac, or PBS. oVV and oVV-Smac $\left(2 \times 10^{7}\right.$ plaque forming unit/mouse) was injected once every day for a total of 4 times through intratumoral injection; gemcitabine was injected intraperitoneally at a total dose of $30 \mathrm{mg} / \mathrm{kg}$ body weight; and PBS $100 \mu \mathrm{l}$ as control for a total of 4 times once every day.

Mice were sacrificed at 2 weeks post-injection according to ethical instructions by carbon dioxide. Tumors were separated, fixed using $4 \%$ paraformaldehyde, embedded in paraffin, finally cut into $4-\mu \mathrm{m}$ sections for hematoxylin and eosin staining, immunohistochemical analysis and TUNEL assay according to the manufacturers' instructions. For immunohistochemical analysis, slides were incubated with primary antibody anti-Smac (dilution 1:100; cat. no. ab8114; Abcam, Shanghai, China) overnight at $4{ }^{\circ} \mathrm{C}$, and then incubated with biotinylated secondary antibody (dilution 1:1,000; cat. no. B2763; Thermo Fisher Scientific, Inc.) and further visualized using a diaminobenzidine (DAB) kit (Thermo Scientific, Inc.).

Statistical analysis. For comparison between 2 groups, significant differences were determined using the Student's t-test. Analysis of variance (ANOVA) followed by a Bonferroni multiple-group comparison test was applied for comparison of 3 or more groups. The analysis of the combined effects was performed with CalcuSyn software 2.0 (Biosoft, Cambridge, UK). Data are expressed as the mean \pm SD. Statistical analysis was performed with IBM SPSS Statistics software version 20 (IBM Corp., Armonk, NY, USA). Statistical significance was defined at $\mathrm{P}<0.05$.

\section{Results}

Characterization of $o V V$-Smac in vitro. The generation of oVV-Smac was performed by homologous recombination as described in our previous study (24). Fig. 1A depicts the construction scheme of oVV and oVV-Smac. Real-time quantitative polymerase chain reaction (qPCR) confirmed exogenous Smac expression. Three pancreatic cancer cell lines, namely PANC-1, SW1990 and BxPC-3, were infected with oVV and oVV-Smac at multiplicities of infection (MOI) of 10 for $24 \mathrm{~h}$. As expected, a significant amount of Smac was observed in all oVV-Smac-transfected pancreatic cancer cell lines, but not in the oVV- or phosphase-buffered saline (PBS)-treated group (Fig. 1B). Similar results were obtained when the expression of Smac and vaccinia virus A27L was determined at the protein level by western blot analysis (Fig. 1C), suggesting that Smac was overexpressed in oVV-Smac-transfected cells both at the transcriptional and translational levels.

To control for any interference of the transgene and modified genome of vaccinia virus with the selective replicative ability of the recombinant oVV in different cell lines, a progeny assay was performed by infecting the three pancreatic cancer cells with various constructs including vaccinia virus (wild-type), oVV and oVV-Smac. The results indicated that both oncolytic viruses oVV and oVV-Smac replicated easily in the infected pancreatic cancer cells and yielded a high virus progeny (Fig. 1D). Thus, the selective replicative ability of oVV in cancer cells was not affected by the insertion of Smac and thymidine kinase (TK) deletion. 
A

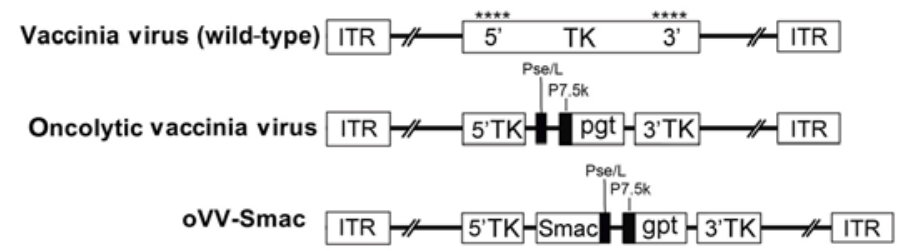

C

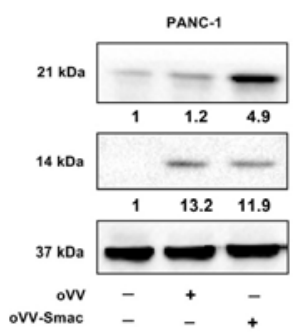

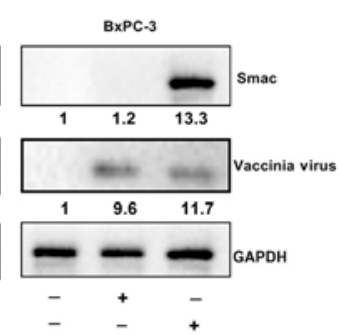
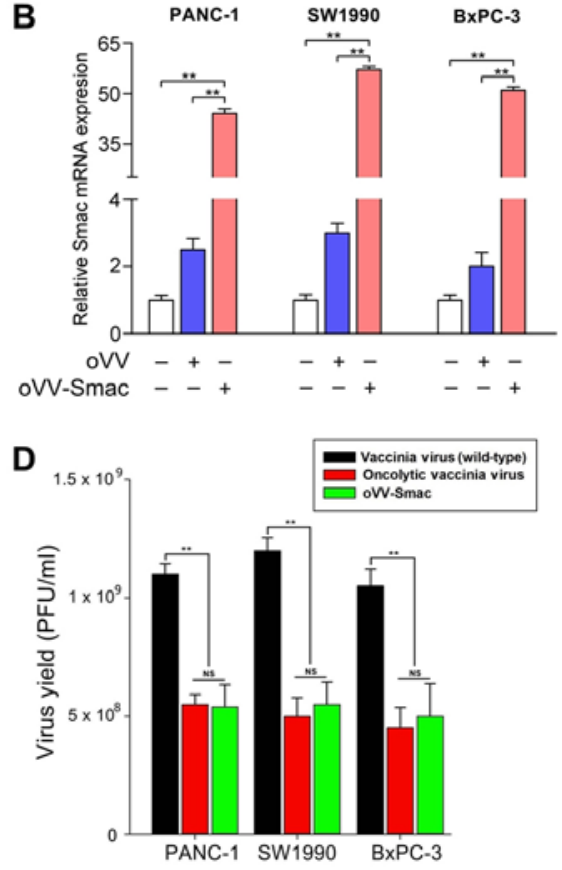

Figure 1. Characterization of oVV-Smac. (A) Schematic structure of Vaccinia virus (wild-type), oncolytic vaccinia virus (oVV) and Smac-armed oncolytic vaccinia virus (oVV-Smac). gpt works as a screen gene. ${ }^{* * * * *}$, sites of anticipated homologous recombination. 5'TK and 3'TK, viral flanking sequences of the thymidine kinase (TK) gene. ITR, inverted terminal repeat. (B) Expression of Smac. Cells were infected with oVV or oVV-Smac at a MOI of 10 for $24 \mathrm{~h}$. The Smac expression was determined using qPCR. GAPDH served as an internal control. Data are presented as mean \pm standard deviation (SD) and are representative of three separate experiments $\left({ }^{* *} \mathrm{P}<0.001\right)$. (C) Detection of expression of Vaccinia virus A27L and Smac by western blot analysis. SW1990 cells were treated with oVV or oVV-Smac at a MOI of 10. After $24 \mathrm{~h}$, cell lysates were prepared for analyzing the expression of Smac and VacciniaVirus proteins. Mock-infected cells were included as a control. GAPDH was used as a protein loading control. (D) Cells ( $\left.2 \times 10^{5}\right)$ were plated into 6 -well plates. After 24 h, the cells were infected with 10 MOIs of oVV-Smac or oVV or vaccinia virus (wild-type), respectively. After an additional 48 h, medium and cells were scraped into a 1.5-ml Eppendorf tube and subjected to three-thaw cycles. The collected supernatant was tested for virus production by standard TCID50 assay on $293 \mathrm{~A}$ cells. Progeny viruses from 1 MOI of virus were calculated. Data are presented as mean \pm standard deviation (SD) and are representative of three separate experiments $\left({ }^{* *} \mathrm{P}<0.01\right.$, NS represents not significance at $\left.\mathrm{P}>0.05\right)$.

In vitro antitumor activity of oVV-Smac. The 3-(4,5-dimethylthiazol-2-yl)-2,5-diphenyltetrazolium bromide (MTT) assay was performed $48 \mathrm{~h}$ post-infection to evaluate the cytotoxicity of oVV-Smac in the PANC-1, SW1990 and BxPC-3 cell lines. The results indicated a significantly higher inhibition of cell growth with oVV-Smac than with oVV (Fig. 2A and B); these effects were dose-dependent. Taken together, these findings demonstrated selective inhibitory effects of oVV-Smac on cancer cell growth in vitro.

Infection with oVV-Smac induces apoptosis in vitro. To address the underlying mechanism of oVV-Smac-induced cytotoxicity, we evaluated oVV-Smac-associated apoptosis in vitro using flow cytometric analysis. The results indicated significant apoptosis in the PANC-1, SW1990 and BxPC-3 cell lines transfected with oVV-Smac compared with the percentage of apoptosis in the oVV- or PBS-treated cells (Fig. 3A).

We further evaluated apoptosis by assessing the expression of apoptosis-related proteins in SW1990 cells at $48 \mathrm{~h}$ post-infection using western blotting analysis. The results indicated a significant activation of caspase-3, -8 and -9, and increased poly(ADP-ribose) polymerase (PARP) cleavage in the oVV-Smac-treated cells (Fig. 3B; left blot). Additionally, the levels of X-linked IAP (XIAP), cellular IAP-1 (cIAP-1), cIAP-2, survivin and livin were also decreased in the oVV-Smac cells (Fig. 3B; right blot). Taken together, these findings indicated that oVV-Smac effectively induced apoptosis through the caspase and IAP pathways.

Combined treatment with gemcitabine and oVV-Smac results in synergistic effects. To determine whether oVV-Smac enhances the cytotoxic effect of gemcitabine, we analyzed the viability of pancreatic cancer cells using the MTT assay after co-treatment with oVV-Smac and gemcitabine. The PANC-1, SW1990 and BxPC-3 cells were treated with gemcitabine (1 or $5 \mu \mathrm{M})$ with or without oVV-Smac $(0.1$ or $0.5 \mathrm{MOI}$ ). The combination of oVV-Smac with gemcitabine significantly inhibited cell growth compared with treatment with gemcitabine or oVV-Smac alone (Fig. 4A upper panels). Next, the synergistic effect of gemcitabine combined with oVV-Smac on pancreatic cancer cells was quantified using the combination index (CI) analysis and expressed as CI vs. the fractional affect (Fig. 4A lower panels). In PANC-1 cells, at all the fractions considered, the Chou-Talalay CI was lower than one $\left(\log _{10}(\mathrm{CI})<0\right)$, indicating a potentiation effect of oVV-Smac when combined with gemcitabine, and vice versa. Additionally, investigation with SW1990 and BxPC-3 cells presented similar results $\left(\log _{10}(\mathrm{CI})<0\right)$. These results showed that the combination of gemcitabine and oVV-Smac had a synergistic tumor killing effect.

We further evaluated apoptosis using Annexin-V-FITC/PI double staining to evaluate the effect of oVV-Smac on gemcitabine-induced apoptosis (Fig. 4B). The apoptotic rate 
A
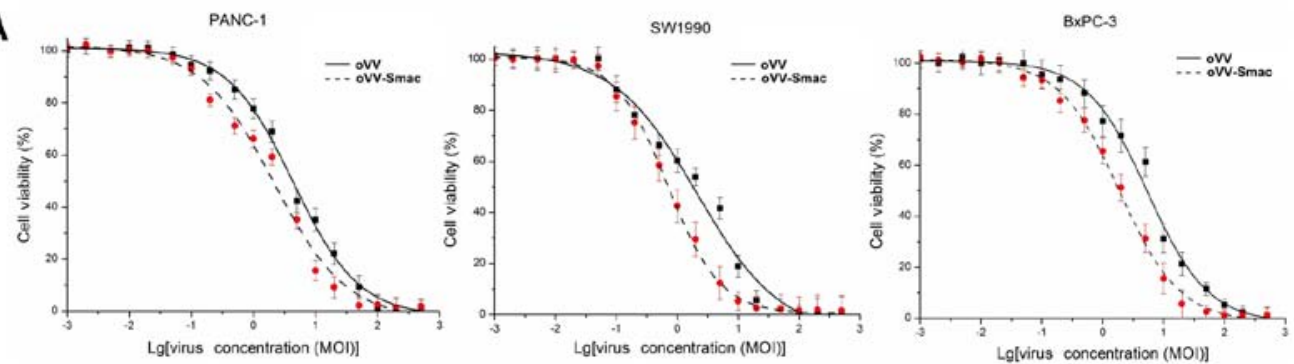

B IC ${ }^{50}$ value

\begin{tabular}{|c|c|c|c|}
\hline Cell line & $\mathrm{IC}_{50}$ oVV & $\mathrm{IC}_{50}$ oVV -Smac & $\begin{array}{l}\text { Fold decrease } \\
\text { Vs. oVV }\end{array}$ \\
\hline PANC-1 & 3.992 & 1.93 & $2.07 \times$ \\
\hline SW1990 & 1.599 & 0.761 & $2.1 \times$ \\
\hline BxPC-3 & 5.043 & 1.758 & $2.87 \times$ \\
\hline
\end{tabular}

Figure 2. Pancreatic cancer-specific cytotoxicity of oVV-Smac. Cells were seeded in 96-well plates and infected with oVV or oVV-Smac at a series of MOIs. Cell viability was determined by MTT cell proliferation assay at $48 \mathrm{~h}$ post-infection. (A) Cytotoxicity and (B) $\mathrm{IC}_{50}$ of oVV and oVV-Smac in three pancreatic cancer cell lines. The results are presented as mean $\pm \mathrm{SD}$ of three separate experiments. oVV, oncolytic vaccinia virus; oVV-Smac, Smac-armed oncolytic vaccinia virus.

A

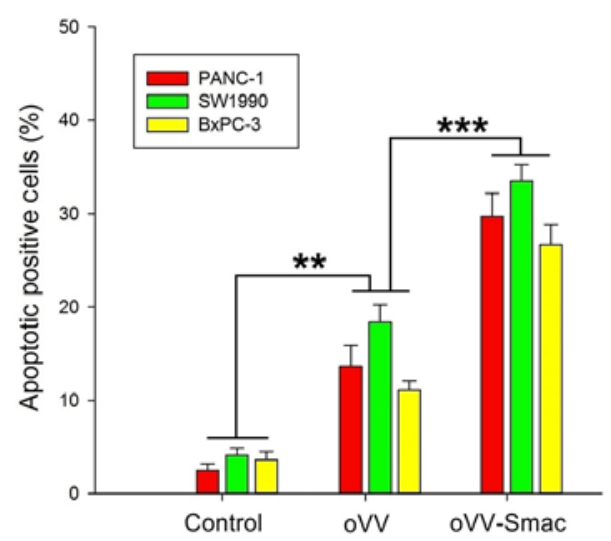

B

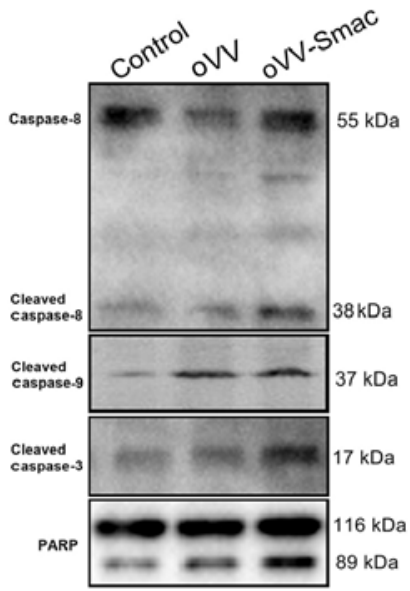

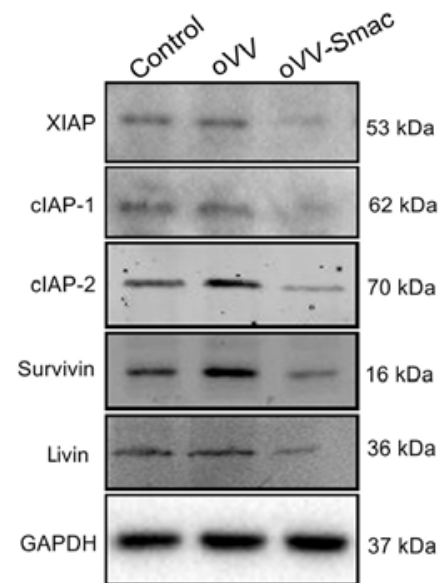

Figure 3. oVV-Smac induces apoptosis in pancreatic cancer cells in vitro. (A) Apoptosis analysis using Annexin V-FITC/PI double staining. SW1990 cells were infected with oVV or oVV-Smac $(\mathrm{MOI}=5)$ for $24 \mathrm{~h}$. The florescence was analyzed by flow cytometry. Data are presented as mean \pm SD of three separate experiments. ${ }^{* *} \mathrm{P}<0.01,{ }^{* * *} \mathrm{P}<0.001$. (B) SW1990 cells were infected with oVV-Smac or oVV (5 MOI) for 24 h. Whole cell extracts were prepared and immunoblotted for the detection of activation of caspase and IAP pathway. GAPDH was used as a loading control. oVV, oncolytic vaccinia virus; oVV-Smac, Smac-armed oncolytic vaccinia virus.

in the pancreatic cancer cells co-treated with gemcitabine and oVV-Smac was significantly higher than that in the cells treated with gemcitabine or oVV-Smac alone.

The question remains as to the mechanism by which oVV-Smac may influence gemcitabine-resistant pancreatic cancercell lines. Therefore, we constructed a gemcitabine-resistant SW1990 cell line (SW1990/GEM). Subsequently, the expression of multidrug resistance-related proteins and IAPs in SW1990/GEM cells treated with oVV-Smac, Smac, or oVV was assessed by western blot analysis. Compared with the sensitive SW1990 cells, SW1990/GEM cells produced marked multidrug resistance-related proteins (P-gp and MDR1) and IAPs (XIAP, cIAP-1 and cIAP-2). Based on these results, Smac gene transfection has the capacity to reduce multidrug 
A

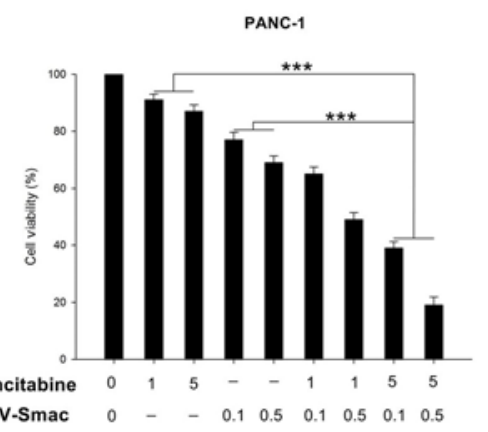

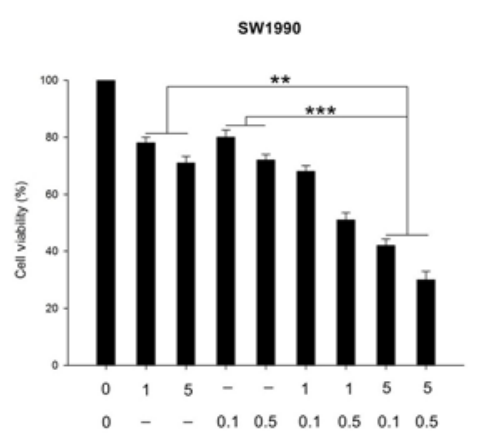

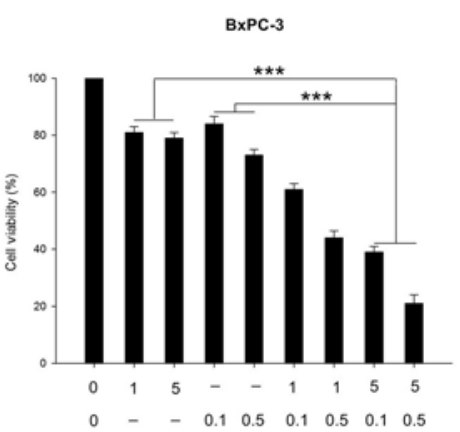

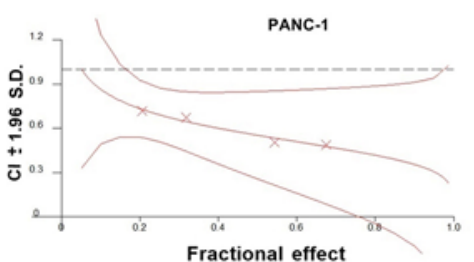

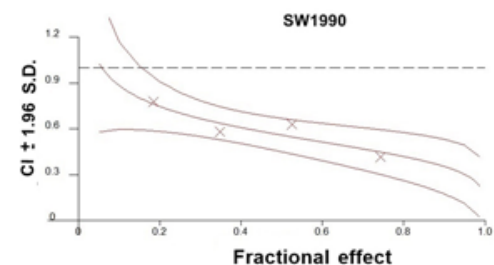

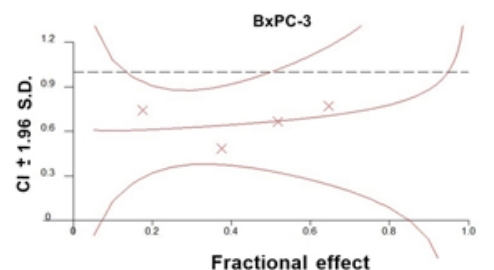

Fractional effect
B

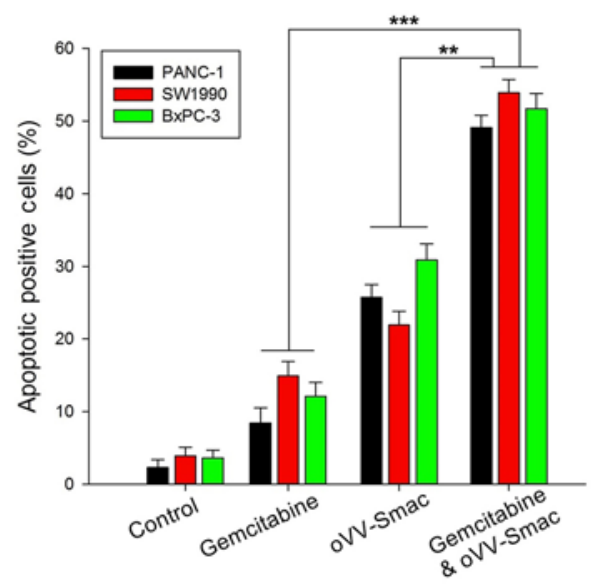

C
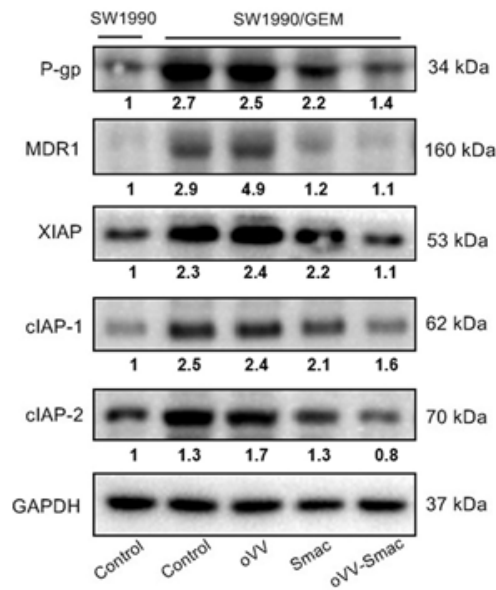

Figure 4.oVV-Smac enhances gemcitabine-mediated growth inhibition and apoptosis in pancreatic cancer cells. (A) Cells were treated with gemcitabine and/or $\mathrm{oVV}$-Smac for $48 \mathrm{~h}$, and cell viability was determined by MTT assay. The potential synergistic effect of gemcitabine combined with oVV-Smac or gemcitabine alone on pancreatic cancer cells. It was assessed by Chou-Talalay Combination Index (CI) analysis using CalcuSyn software. The middle curve line stands for the simulated combination index values, which was expressed as the $\log _{10}(\mathrm{CI}) \pm 1.96 \mathrm{SD}$, encircled by two lines of algebraic evaluation of the $95 \%$ confidence intervals. The $\log _{10}(\mathrm{CI})$ values attained at the given fractional effects represent an antagonism between the treatments when $>0$, an additive efficiency when equal to 0 and asynergism when $<0$. It was quantified by $\mathrm{CI}$ analysis and expressed as $\mathrm{CI}$ vs. the fraction affected. Where calculable, $95 \%$ confidence intervals are shown. (B) Gemcitabine $(5 \mu \mathrm{g} / \mathrm{ml})$, oVV-Smac $(0.1 \mathrm{MOI})$, or gemcitabine $(5 \mu \mathrm{g} / \mathrm{ml})$ plus oVV-Smac $(0.1 \mathrm{MOI})$ was used to treat PANC-1, SW1990 and BxPC-3 cells. Uninfected cells served as the control. Forty-eight hours later, apoptosis was determined by flow cytometry. (C) Gemcitabine-resistant SW1990 cell line (SW1990/GEM) was used to elucidate the mechanism by which oVV-Smac may influence gemcitabine-resistant pancreatic cancer. oVV-Smac (10 MOI), oVV (10 MOI), or Smac (5 $\mu \mathrm{g})$ was used to treat SW1990/GEM cells. Uninfected cells served as the control. Forty-eight hours later, whole cell extracts were prepared and immunoblotted. GAPDH was used as a loading control. ( ${ }^{* *} \mathrm{P}<0.01$ and ${ }^{* * * *} \mathrm{P}<0.001$, one-way analysis of variance (ANOVA) and multiple comparisons). oVV, oncolytic vaccinia virus; oVV-Smac, Smac-armed oncolytic vaccinia virus.

resistance-related proteins and IAPs, which could be further potentiated by Smac-armed oncolytic vaccinia virus (Fig. 4C).

Taken together, these findings indicated a synergistic repressive effect of the combined gemcitabine and oVV-Smac treatment on pancreatic cancer cell proliferation.

Enhanced cytotoxic effect of co-treatment with gemcitabine and oVV-Smac in vivo. We developed two pancreatic tumor xenograft mouse models with sensitive SW1990 cells and SW1990/GEM cells using BALB/c athymic nude mice to evaluate the effect of co-treatment with gemcitabine and oVV-Smac in vivo (Fig. 5A). Antitumor efficacy was evaluated by plotting tumor growth curves over a 56-day observation period. The mean tumor volume was significantly decreased in mice injected with gemcitabine, oVV-Smac, and both compared with those injected with PBS (Fig. 5B and C). Furthermore, co-treatment of sensitive SW1990 and SW1990/GEM cells with gemcitabine and oVV-Smac was more effective than gemcitabine $(\mathrm{P}=0.001$ and 0.002 , respectively) and oVV-Smac alone $(\mathrm{P}=0.001$ and 0.003 , respectively). Co-treatment with gemcitabine and oVV-Smac was also associated with a higher survival rate when compared with treatment with PBS, gemcitabine, or oVV-Smac (Fig. 5D and E). 
A

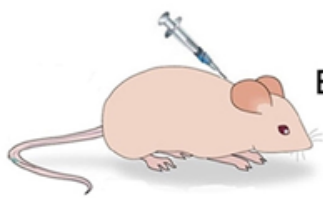

Day 0

BALB/c nude, male, 6-week-old mice

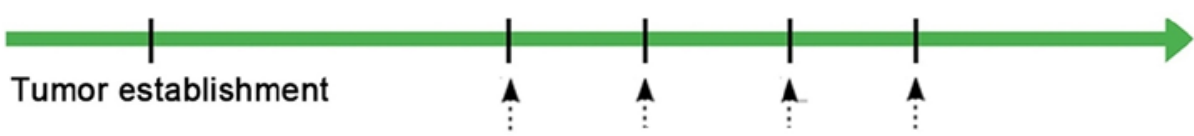

SW1990 or SW1990/GEM
cell injection

Day 15 (first day of injection) average tumor volume: $100 \mathrm{~mm}^{3}$

B
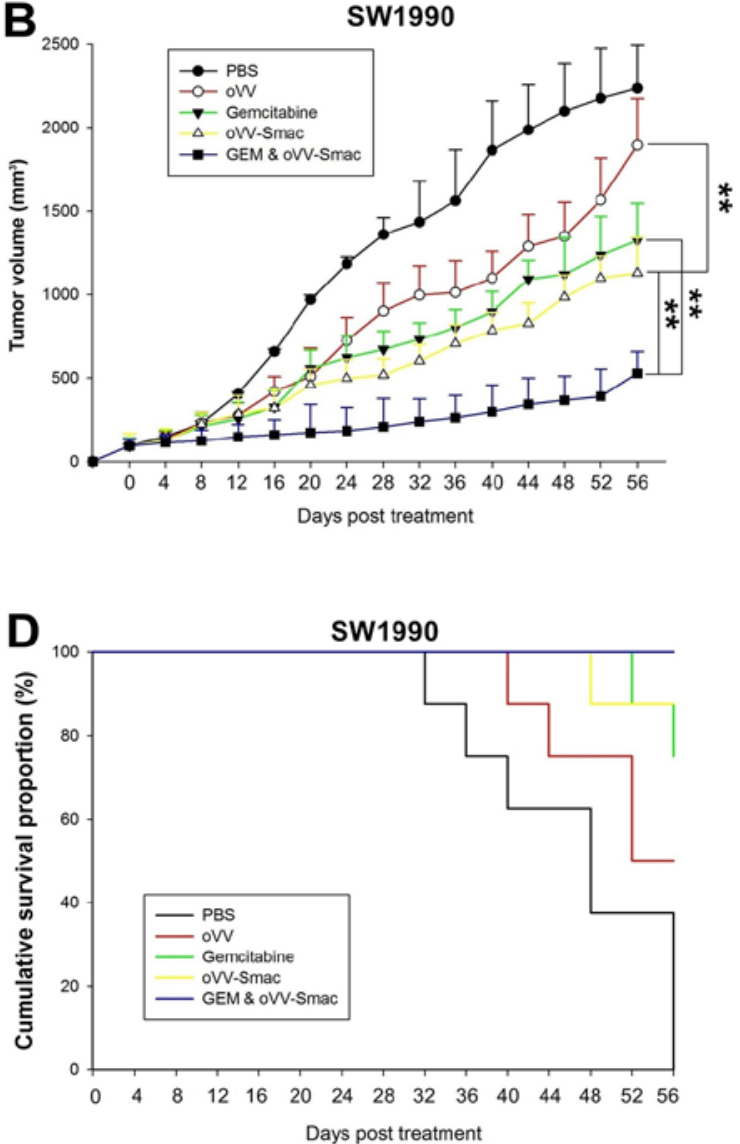
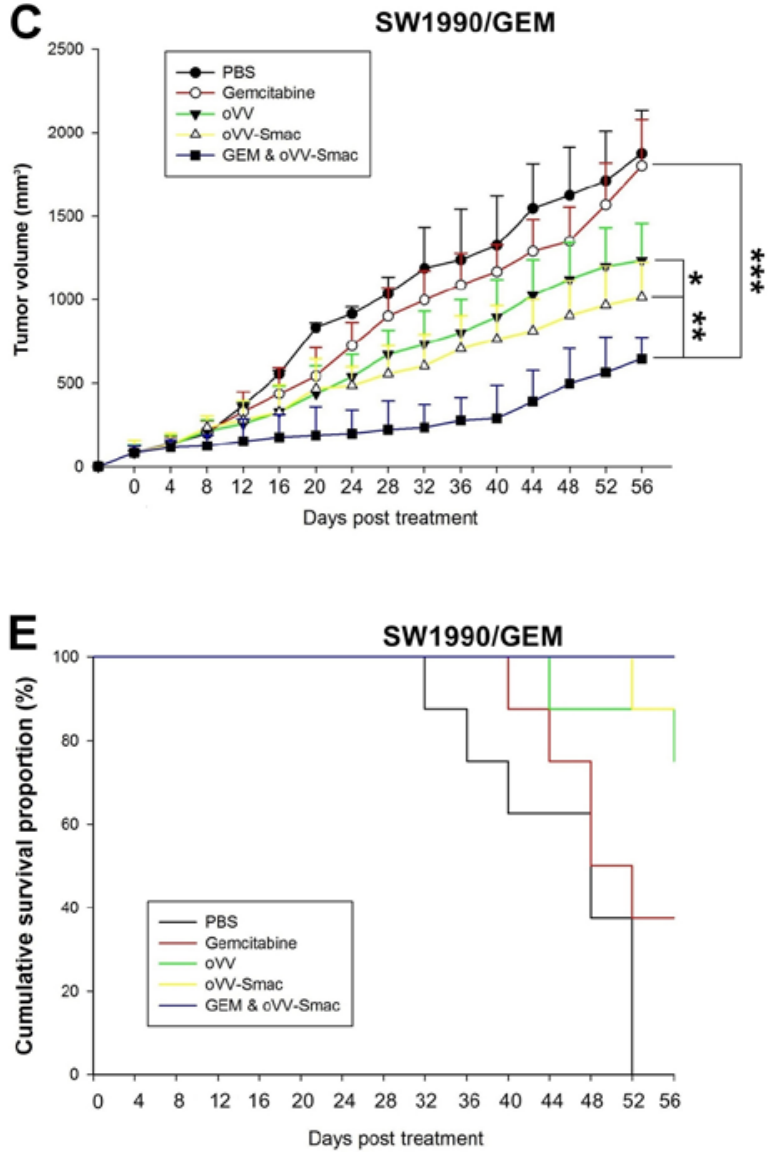

Figure 5. Synergistic effects of gemcitabine and oVV-Smac in vivo. (A) Schematic diagram of injections with the experimental timeline. BALB/c athymic nude mice with SW1990 or SW1990/GEM tumor xenografts were intratumorally injected with PBS (100 $\mu 1)$, oVV (2x10 ${ }^{7}$ pfu) or oVV-Smac ( $2 \times 10^{7}$ pfu) every day for a total of 4 times, or intraperitoneally injected with a single dose of gemcitabine $(50 \mathrm{mg} / \mathrm{kg})$ every day for 4 times or a combination therapy based on gemcitabine and oVV-Smac. (B and C) The tumor volume was measured at different times after treatment. Data are presented as mean \pm standard error $(\mathrm{n}=8$ ) ${ }^{*} \mathrm{P}<0.05,{ }^{* *} \mathrm{P}<0.01$ and ${ }^{* * *} \mathrm{P}<0.001$, one-way analysis of variance (ANOVA) and multiple comparisons. (D and E) The images show the inhibitory effect of each treatment group on tumor growth at the last time point (day 56) when the mice were sacrificed. GEM, gemcitabine; oVV, oncolytic vaccinia virus; oVV-Smac, Smac-armed oncolytic vaccinia virus.

The tumor histopathological changes were further evaluated by hematoxylin and eosin (H\&E) staining, immunohistochemistry (IHC) and TUNEL assay. The combined treatment with gemcitabine and oVV-Smac resulted in a higher cytotoxicity than single treatment as evidenced by H\&E staining. Moreover, an intense expression of Smac in the tumor tissues was associated with the combined treatment as evidenced by IHC with anti-Smac (Fig. 6). Apoptosis was further examined using the terminal deoxynucleotidyl transferase-mediated dUTP nick end labeling (TUNEL) assay. Results from this experiment showed significantly higher apoptosis rates in the combination treatment when compared with either individual treatment (Fig. 6). 


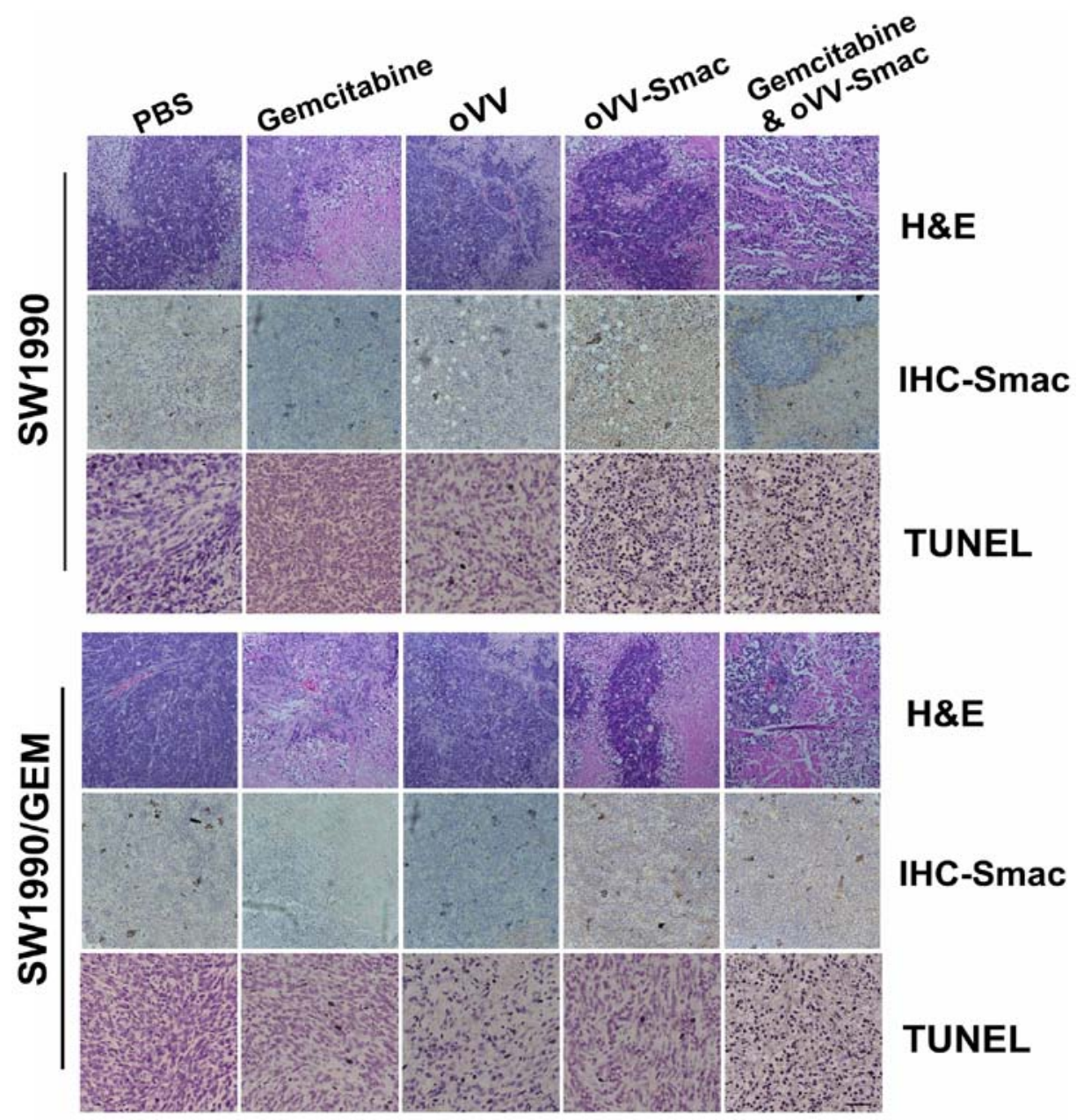

Figure 6. Histopathological analysis. Tumor samples from the different groups were subjected to H\&E staining, immunohistochemical and TUNEL analysis. Magnification, x200. oVV, oncolytic vaccinia virus; oVV-Smac, Smac-armed oncolytic vaccinia virus.

\section{Discussion}

Considering the genetic diversity of most tumors and the development of drug-resistance, monotherapy has been associated with limited success in various types of cancer (26). Therefore, the identification of therapeutic agents that act synergistically when combined through different mechanisms without inducing adverse effects is critical in cancer therapy (27). Many cancer cells express elevated levels of inhibitor of apoptosis proteins (IAPs) and escape apoptosis through the activity of IAPs (28). IAPs prevent the activation of caspases and, as such, block the extrinsic and intrinsic apoptotic cascades. XIAP is one of the best characterized IAPs and is expressed at a higher level in pancreatic cancer cell lines and pancreatic tumors compared with normal pancreas (29). The mitochondrial protein Smac inhibits IAPs, including XIAP, thus promoting caspase activation and subsequent cell death. Smac has been shown to bind to XIAP, cIAP-1 and cIAP-2, and Smac mimetics sensitize tumors to programmed cell death. In the present study, Smac exhibited low expression in our pancreatic cancer cell lines (PANC-1, SW1990 and BxPC-3), which was confirmed in our previous study (21). In addition, Smac is expressed at a lower level in pancreatic tumors (10 of 10) compared with normal pancreas (data not shown). Several clinical studies have reported synergistic antitumor activity of the co-treatment with oVV and chemotherapy or radiation therapy $(30,31)$. Additionally, it has been suggested that the route of combination of oVV with cytotoxic chemotherapies is another factor in potentiating the effects of oVV and optimizing the therapy (32).

Pancreatic cancer is invariably aggressive with high mortality rates, while the acquired drug resistance makes it intransigent (33). Thus, new effective therapeutic approaches are urgently required. Gene therapy has now become an innovative approach for cancer treatment. Thus, we developed and evaluated the effect of oVV-based gene therapy in vitro and in vivo. The results indicated significant growth inhibition with relatively limited side effects.

Interestingly, the mitochondrial protein Smac is released into the cytosol during the process of apoptosis. Recently, Smac has been reported to exhibit significant cytotoxic effects on different tumor types (34). In addition, Smac was found to potentiate the sensitivity to chemotherapeutic drugs in patients with cancer, indicating that the targeted delivery of Smac may be an auspicious gene therapy in pancreatic cancer (35). However, no studies have reported on oVV-mediated Smac gene therapy in pancreatic cancer. We developed a novel oVV (TK deletion) that expresses Smac (oVV-Smac), and assessed its antitumor effect when applied alone or combined with gemcitabine. 
Based on our results, oVV-Smac potentiated apoptosis in human pancreatic cancer cells. The IAPs bind to caspases and inhibit their activity through their baculovirus IAP repeat domains (36). Furthermore, IAPs may ubiquitinate themselves and their interacting proteins through the ubiquitin-protein isopeptide ligase activity of their Really interesting new gene (RING) finger domain (37). On the other hand, Smac serves as a key molecule in reducing the protein levels of XIAP, c-IAPs, survivin and livin both in vitro and in vivo through the ubiquitin/proteasome pathway. In addition, Smac is implicated in mitochondrial apoptosis pathways and promotes chemotherapy-induced apoptosis $(38,39)$. Our present findings demonstrated a significant reduction of IAPs and activation of caspase-3, -8, -9 and PARP in SW1990 pancreatic cancer cells transfected with oVV-Smac. These results suggested the implication of caspase-dependent apoptosis and IAPs in oVV-Smac-induced cytotoxicity.

Our results also demonstrated synergistic cytotoxic effects of co-treatment with oVV-Smac and gemcitabine both in vitro and in vivo. Several mechanisms may account for this synergistic effect (40). First, gemcitabine, which is a first-line intervention in pancreatic cancer, bears the limitation of weak penetration into the tumor parenchyma. In contrast, oVV-Smac selectively replicates in tumor cells, thus resulting in their lysis, which disrupts the tumor's architecture and facilitates the penetration of gemcitabine. Thus, gemcitabine combined with oVV-Smac result in a synergistic effect. Second, gemcitabine was found to enhance vaccine efficacy by eliminating $\mathrm{CD} 11 \mathrm{~b}^{+} / \mathrm{Gr}-1^{+}$myeloid-derived suppressor cells (MDSCs) in a murine model of pancreatic carcinoma, which may be another mechanism for the synergy $(41,42)$. Third, suboptimal doses of gemcitabine have been reported to stimulate the viral uptake in pancreatic cancer cells, which may explain the observed synergistic effects. Fourth, both oVV-Smac and gemcitabine induce apoptosis, which may explain their synergistic effects (43). Finally, unblocking host pathways, transporting viruses with greater efficiency, and/or increasing viral replication at the tumor site may also account for the synergistic effects of oVV-Smac and gemcitabine when combined.

To the best of our knowledge, this is the first report concerning the cytotoxic effects of oVV-Smac on pancreatic cancer cells and the synergistic effects of co-treatment with oVV-Smac and gemcitabine. Undoubtedly, the optimization of oVV-Smac application in clinical practice is warranted, particularly in relation to increasing the delivery and expression of Smac, viral vector cytotoxicity, and immune response to viral antigens. Thus, additional research aimed at promoting the transfection efficiency of oVV-Smac and reducing its potential toxicity is required.

\section{Acknowledgements}

Not applicable.

\section{Funding}

The present study was supported by the National Science Foundation of China (nos. 81602706 and 81570198), the Funds of Science Technology Department of Zhejiang
Province (no. 2017C33116), the Zhejiang Medical Technology Plan Project (no. 2016KYA018) and State Administration of Traditional Chinese Medicine of Zhejiang (no. 2017ZB006).

\section{Availability of data and materials}

The datasets used during the present study are available from the corresponding author upon reasonable request.

\section{Authors' contributions}

SW, GR and XM conceived and designed the study. WC, WF, XZ, FH, XL and SW performed the experiments. SW, WC and GR wrote the paper. SW, XL, XZ and WC reviewed and edited the manuscript. All authors read and approved the manuscript and agree to be accountable for all aspects of the research in ensuring that the accuracy or integrity of any part of the work are appropriately investigated and resolved.

\section{Ethics approval and consent to participate}

All animal experiments were approved by the Institutional Animal Care and Use Committee, Zhejiang Provincial People's Hospital and all procedures were in according to the Guide for the Care and Use of Laboratory Animals (National Academies Press, Washington, D.C.).

\section{Patient consent for publication}

Not applicable.

\section{Competing interests}

The authors declare that they have no competing interests.

\section{References}

1. Ryan DP, Hong TS and Bardeesy N: Pancreatic adenocarcinoma. N Engl J Med 371: 1039-1049, 2014.

2. Bednar F and Simeone DM: Recent advances in pancreatic surgery. Current Opin Gastroenterol 30: 518-523, 2014.

3. Ghaneh P,Smith R, Tudor-Smith C, Raraty Mand Neoptolemos JP: Neoadjuvant and adjuvant strategies for pancreatic cancer. Eur J Surg Oncol 34: 297-305, 2008.

4. Vaccaro V, Sperduti I and Milella M: FOLFIRINOX versus gemcitabine for metastatic pancreatic cancer. N Engl J Med 365: 768-769, 2011.

5. Kristensen A, Vagnildhaug OM, Gronberg BH, Kaasa S, Laird B and Solheim TS: Does chemotherapy improve health-related quality of life in advanced pancreatic cancer? A systematic review. Crit Rev Oncol Hematol 99: 286-298, 2016.

6. Sultana A, Smith CT, Cunningham D, Starling N, Neoptolemos JP and Ghaneh P: Meta-analyses of chemotherapy for locally advanced and metastatic pancreatic cancer. J Clin Oncol 25: 2607-2615, 2007.

7. Kirn DH and Thorne SH: Targeted and armed oncolytic poxviruses: A novel multi-mechanistic therapeutic class for cancer. Nat Rev Cancer 9: 64-71, 2009.

8. Kaufman HL, Deraffele G, Mitcham J, Moroziewicz D, Cohen SM, Hurst-Wicker KS, Cheung K, Lee DS, Divito J, Voulo M, et al: Targeting the local tumor microenvironment with vaccinia virus expressing B7.1 for the treatment of melanoma. J Clin Invest 115: 1903-1912, 2005.

9. Chard LS, Lemoine NR and Wang Y: New role of Interleukin-10 in enhancing the antitumor efficacy of oncolytic vaccinia virus for treatment of pancreatic cancer. Oncoimmunology 4: e1038689, 2015. 
10. Liu Z, Ravindranathan R, Li J, Kalinski P, Guo ZS and Bartlett DL: CXCL11-armed oncolytic poxvirus elicits potent antitumor immunity and shows enhanced therapeutic efficacy. Oncoimmunology 5: e1091554, 2015.

11. Heo J, Reid T, Ruo L, Breitbach CJ, Rose S, Bloomston M, Cho M, Lim HY, Chung HC, Kim CW, et al: Randomized dose-finding clinical trial of oncolytic immunotherapeutic vaccinia JX-594 in liver cancer. Nat Med 19: 329-336, 2013.

12. Breitbach CJ, Thorne SH, Bell JC and Kirn DH: Targeted and armed oncolytic poxviruses for cancer: The lead example of JX-594. Curr Pharm Biotechnol 13: 1768-1772, 2012.

13. Deng L, Fan J, Ding Y, Zhang J, Zhou B, Zhang Y and Huang B Oncolytic efficacy of thymidine kinase-deleted vaccinia virus strain Guang9. Oncotarget 8: 40533-40543, 2017.

14. Zhang Q, Yu YA, Wang E, Chen N, Danner RL, Munson PJ, Marincola FM and Szalay AA: Eradication of solid human breast tumors in nude mice with an intravenously injected light-emitting oncolytic vaccinia virus. Cancer Res 67: 10038-10046, 2007.

15. Park SH, Breitbach CJ, Lee J, Park JO, Lim HY, Kang WK, Moon A, Mun JH, Sommermann EM, Maruri Avidal L, et al: Phase 1b trial of biweekly intravenous Pexa-Vec (JX-594), an oncolytic and immunotherapeutic vaccinia virus in colorectal cancer. Mol Ther 23: 1532-1540, 2015.

16. Kochneva G, Zonov E, Grazhdantseva A, Yunusova A, Sibolobova G, Popov E, Taranov O, Netesov S, Chumakov P and Ryabchikova E: Apoptin enhances the oncolytic properties of vaccinia virus and modifies mechanisms of tumor regression. Oncotarget 5: 11269-11282, 2014.

17. Timiryasova TM, Li J, Chen B, Chong D, Langridge $\mathrm{WH}$, Gridley DS and Fodor I: Antitumor effect of vaccinia virus in glioma model. Oncol Res 11: 133-144, 1999.

18. Ghobrial IM, Witzig TE and Adjei AA: Targeting apoptosis pathways in cancer therapy. CA Cancer J Clin 55: 178-194, 2005

19. Chauhan D and Anderson KC: Mechanisms of cell death and survival in multiple myeloma (MM): Therapeutic implications. Apoptosis 8: 337-343, 2003.

20. Chauhan D, Neri P, Velankar M, Podar K, Hideshima T, Fulciniti M, Tassone P, Raje N, Mitsiades C, Mitsiades N, et al: Targeting mitochondrial factor Smac/DIABLO as therapy for multiple myeloma (MM). Blood 109: 1220-1227, 2007.

21. Ge Y, Lei W, Ma Y, Wang Y, Wei B, Chen X, Ru G, He X, Mou X and Wang S: Synergistic antitumor effects of CDK inhibitor SNS032 and an oncolytic adenovirus co-expressing TRAIL and Smac in pancreatic cancer. Mol Med Rep 15: 3521-3528, 2017.

22. Pan Q, Huang Y, Chen L, Gu J and Zhou X: SMAC-armed vaccinia virus induces both apoptosis and necroptosis and synergizes the efficiency of vinblastine in HCC. Human Cell 27: 162-171, 2014

23. Wang S, Shu J, Chen L, Chen X, Zhao J, Li S, Mou X and Tong X: Synergistic suppression effect on tumor growth of ovarian cancer by combining cisplatin with a manganese superoxide dismutase-armed oncolytic adenovirus. Onco Targets Ther 9: 6381-6388, 2016

24. Lei W, Wang S, Yang C, Huang X, Chen Z, He W, Shen J, Liu X and Qian W: Combined expression of miR-34a and Smac mediated by oncolytic vaccinia virus synergistically promote anti-tumor effects in Multiple Myeloma. Sci Rep 6: 32174, 2016.

25. Buller RM, Smith GL, Cremer K, Notkins AL and Moss B: Decreased virulence of recombinant vaccinia virus expression vectors is associated with a thymidine kinase-negative phenotype. Nature 317: 813-815, 1895 .

26. Levinson AD: Cancer therapy reform. Science 328: 137, 2010.

27. Kaiser J: Combining targeted drugs to stop resistant tumors. Science 331: 1542-1545, 2011.

28. Salvesen GS and Duckett CS: IAP proteins: Blocking the road to death's door. Nat Rev Mol Cell Biol 3: 401-410, 2002.
29. Lopes RB, Gangeswaran R, Mcneish IA, Wang Y and Lemoine NR: Expression of the IAP protein family is dysregulated in pancreatic cancer cells and is important for resistance to chemotherapy. Int J Cancer 120: 2344-2352, 2007.

30. Wilkinson MJ, Smith HG, McEntee G, Kyula-Currie J, Pencavel TD, Mansfield DC, Khan AA, Roulstone V, Hayes AJ and Harrington KJ: Oncolytic vaccinia virus combined with radiotherapy induces apoptotic cell death in sarcoma cells by down-regulating the inhibitors of apoptosis. Oncotarget 7: 81208-81222, 2016.

31. Wu Y, Mou X, Wang S, Liu XE and Sun X: ING4 expressing oncolytic vaccinia virus promotes anti-tumor efficiency and synergizes with gemcitabine in pancreatic cancer. Oncotarget 8 : 82782-82739, 2017.

32. Liu Z, Ravindranathan R, Kalinski P, Guo ZS and Bartlett DL: Rational combination of oncolytic vaccinia virus and PD-L1 blockade works synergistically to enhance therapeutic efficacy. Nat Commun 8: 14754, 2017.

33. Hidalgo M: Pancreatic cancer. N Engl J Med 362: 1605-1617, 2010.

34. Martinez-Ruiz G, Maldonado V, Ceballos-Cancino G, Grajeda JP and Melendez-Zajgla J: Role of Smac/DIABLO in cancer progression. J Exp Clin Cancer Res 27: 1-7, 2008.

35. Fulda S, Wick W, Weller M and Debatin KM: Smac agonists sensitize for Apo2L/TRAIL- or anticancer drug-induced apoptosis and induce regression of malignant glioma in vivo. Nat Med 8: 808-815, 2002

36. Shiozaki EN and Shi Y: Caspases, IAPs and Smac/DIABLO: Mechanisms from structural biology. Trends Biochem Sci 29: 486-494, 2004

37. Bader M and Steller H: Regulation of cell death by the ubiquitin-proteasome system. Curr Opin Cell Biol 21: 878-884, 2009.

38. Xu Y, Zhou L, Huang J, Liu F, Yu J, Zhan Q, Zhang L and Zhao X: Role of Smac in determining the chemotherapeutic response of esophageal squamous cell carcinoma. Clin Cancer Res 17: 5412-5422, 2011.

39. Tirrò E, Consoli ML, Massimino M, Manzella L, Frasca F, Sciacca L, Vicari L, Stassi G, Messina L, Messina A and Vigneri P: Altered expression of c-IAP1, survivin, and Smac contributes to chemotherapy resistance in thyroid cancer cells. Cancer Res 66: 4263-4272, 2006.

40. Metwalli AR, Khanbolooki S, Jinesh G, Sundi D, Shah JB, Shrader M, Choi W, Lashinger LM, Chunduru S, Mcconkey DJ, et al: Smac mimetic reverses resistance to TRAIL and chemotherapy in human urothelial cancer cells. Cancer Biol Ther 10: 885-892, 2010.

41. Ishizaki H, Manuel ER, Song GY, Srivastava T, Sun S, Diamond DJ and Ellenhorn JD: Modified vaccinia Ankara expressing survivin combined with gemcitabine generates specific antitumor effects in a murine pancreatic carcinoma model. Cancer Immunol Immunother 60: 99-109, 2011.

42. Al Yaghchi C, Zhang Z, Alusi G, Lemoine NR and Wang Y: Vaccinia virus, a promising new therapeutic agent for pancreatic cancer. Immunotherapy 7: 1249-1258, 2015.

43. Nguyen TL, Tumilasci VF, Singhroy D, Arguello M and Hiscott J: The emergence of combinatorial strategies in the development of RNA oncolytic virus therapies. Cell Microbiol 11: 889-897, 2009.

This work is licensed under a Creative Commons Attribution-NonCommercial-NoDerivatives 4.0 International (CC BY-NC-ND 4.0) License. 